\title{
Adaptable Parallel Acceleration Strategy for Dynamic Monte Carlo Simulations of Polymerization with Microscopic Resolution
}

\section{-Supporting Information-}

\author{
Rui Liu', Antonios Armaou ${ }^{2,3}$, Xi Chen ${ }^{1,4^{*}}$
}

\begin{abstract}
${ }^{1}$ State Key Laboratory of Industrial Control Technology, College of Control Science and Engineering, Zhejiang University, Hangzhou 310027, China

${ }^{2}$ Chemical Engineering Department, The Pennsylvania State University, PA 16802, USA

${ }^{3}$ Mechanical Engineering Department, The Pennsylvania State University, PA 16802, USA

${ }^{4}$ National Center for International Research on Quality-targeted Process Optimization and Control, China
\end{abstract}

\section{This file includes:}

Section A: Detailed Steps of the DMC Simulation of the Mechanism in Table 2

Section B: Detailed Algorithms

Section C: Data Structure Details

Section D: Sampling Method

Section E: Simulation Metrics

Section F: Comparison with Deterministic Model Results

Section G: Calculation of Microscopic Rates

\footnotetext{
*Correspondence concerning this article should be addressed to $\mathrm{Xi}$ Chen at xi_chen@zju.edu.cn.
} 


\section{Section A. Detailed Steps of the DMC Simulation of the Mechanism in Table 2}

The detailed steps of the DMC simulation of the mechanism presented in Table 2 are summarized as follows:

Step 1. Initialization. Input the parameters, including the number of each reactant species, the size of the reaction system, and the seed for the random number generator. Set the reporting time and target conversion as a simulation stop condition. Initialize the timer and counter.

Step 2. Calculation of the microscopic reaction rates. Calculate the MC microscopic reaction rates for all reaction events at the current state of the system.

Step 3. Calculation of transition probabilities. Calculate the probability for each reaction event by Equation (3).

Step 4. Reaction event selection. Generate a random number from $\mathcal{U}(0,1)$ and select one reaction event by Equation (2).

Step 5. System state update. According to the selected reaction event, update the reactant number. Specifically, when the chain propagation event is selected, generate one random number from $\mathcal{U}(0,1)$ to choose a radical chain and increase the length of this chain by one. When the chain termination event is selected, generate two random numbers from $\mathcal{U}(0,1)$ to choose two radical chains and transform them into dead polymers. Decrease the growing radical number by two and increase the dead polymer number by one. When the chain initiation event is selected, decrease the number of initiators by one and generate one random number from $\mathcal{U}(0,1)$. If the random number is less than the initiation efficiency $f$, increase the number of growing radicals by two; otherwise, go to Step 2.

Step 6. Reaction time update. Generate a random number from $\mathcal{U}(0,1)$, calculate system time step $\tau$ by Equation (1), and advance the timer by $\tau$.

Step 7. Simulation stop criterion. If the required reaction time or conversion is reached, stop the simulation; otherwise, go back to Step 2.

Step 8. Microscopic properties counting. Count all the recorded molecular information in the simulation to obtain the CLD and MWD results. Specifically, during the DMC simulation, the molecular information of the growing radicals and dead polymers is stored in arrays or vectors. The size of the array or vector is the number of growing radicals or dead polymers. The returned index in a searching operation points to one of the growing radicals or dead polymers, and the corresponding element of the index 
is the chain length. If the simulation stops, the specific microscopic properties such as CLD and MWD are obtained through post-processing the data in arrays or vectors.

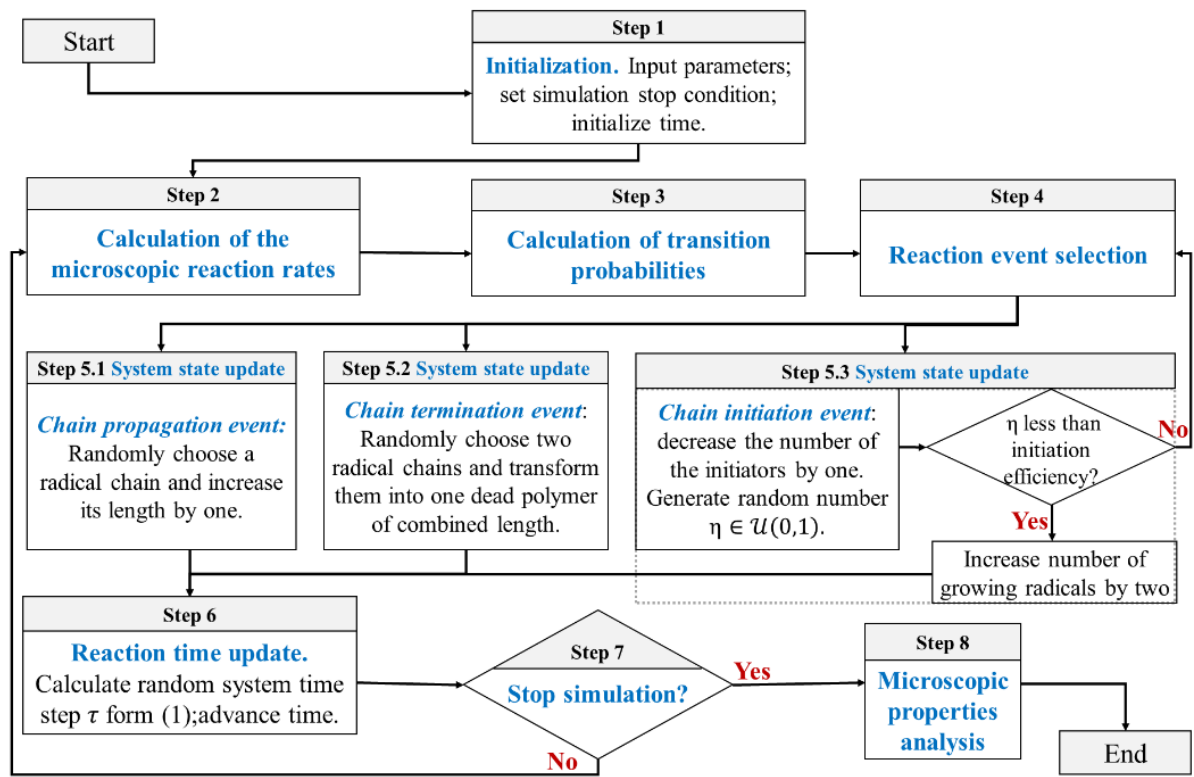

Figure S1. Flowchart for the DMC simulation of the reaction mechanism in Table 2. 


\section{Section B. Detailed Algorithms}

This section presents pseudocodes of the algorithms used to perform the presented simulations. Algorithm S1, presents the DMC simulation for the chain transfer to polymer events. Algorithm S2, details the fork-join parallelization procedure used by OpenMP to perform simulations that involve parameter input, information counting to obtain microscopic properties in serial and system evolution in parallel. The 2D-vector data structure used in OpenMP programming is also presented. Algorithm S3, shows the generation of the random number sequence using the built-in library curand of CUDA and the 1D-array data structure used on CUDA. Algorithm S4 presents the pseudo-code using function cudaOccupancyMaxPotentialBlockSize() to return the optimal block size in the parallel MC method on CUDA. Data communication, kernel function invocation and information recording of Case 1 are detailed in Algorithm S4. 
Algorithm S1. Operations when chain transfer to polymer event is chosen.

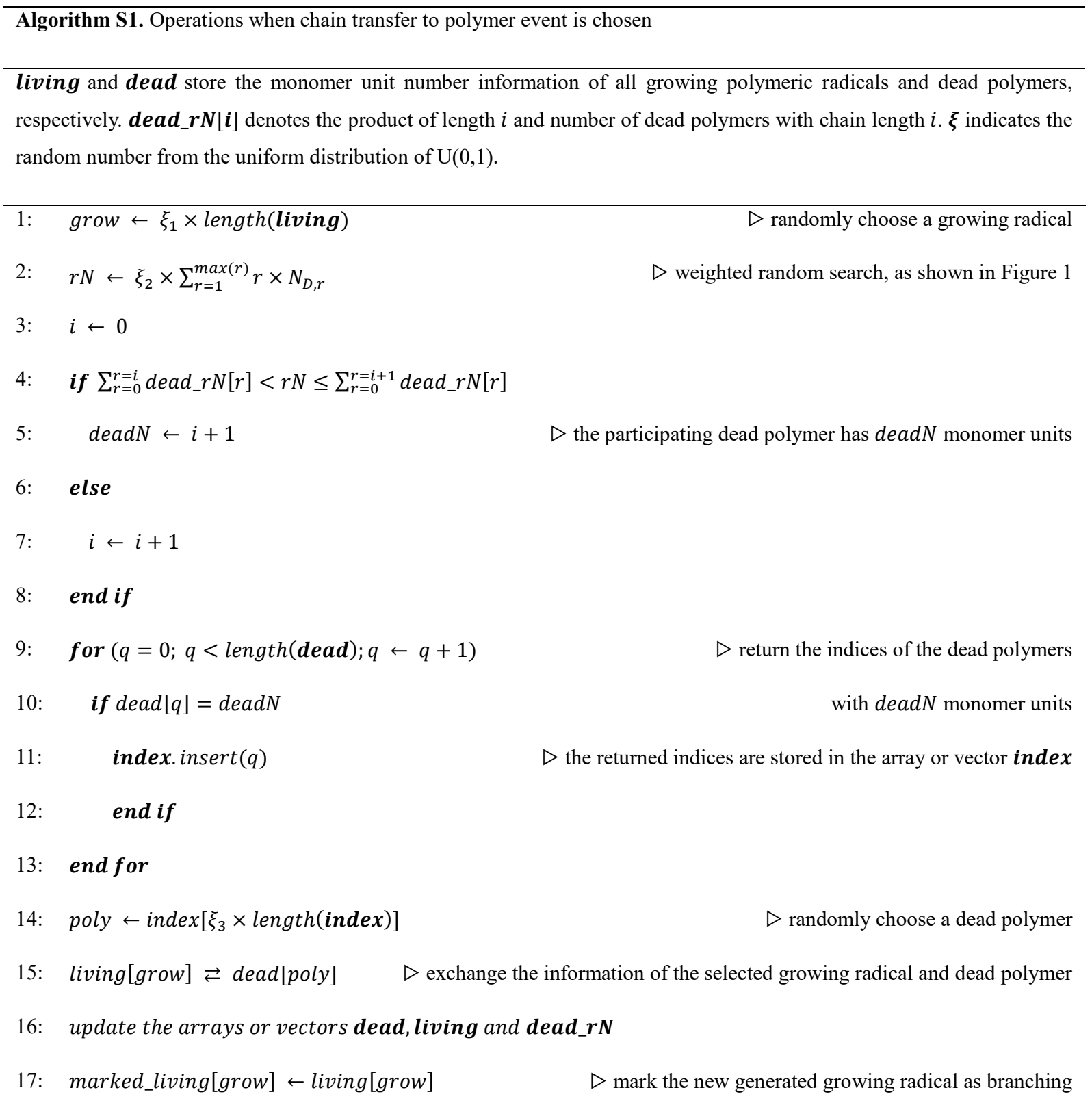


Algorithm S2. The fork-join model and the 2D-vector data structure used in the OpenMP parallel programming.

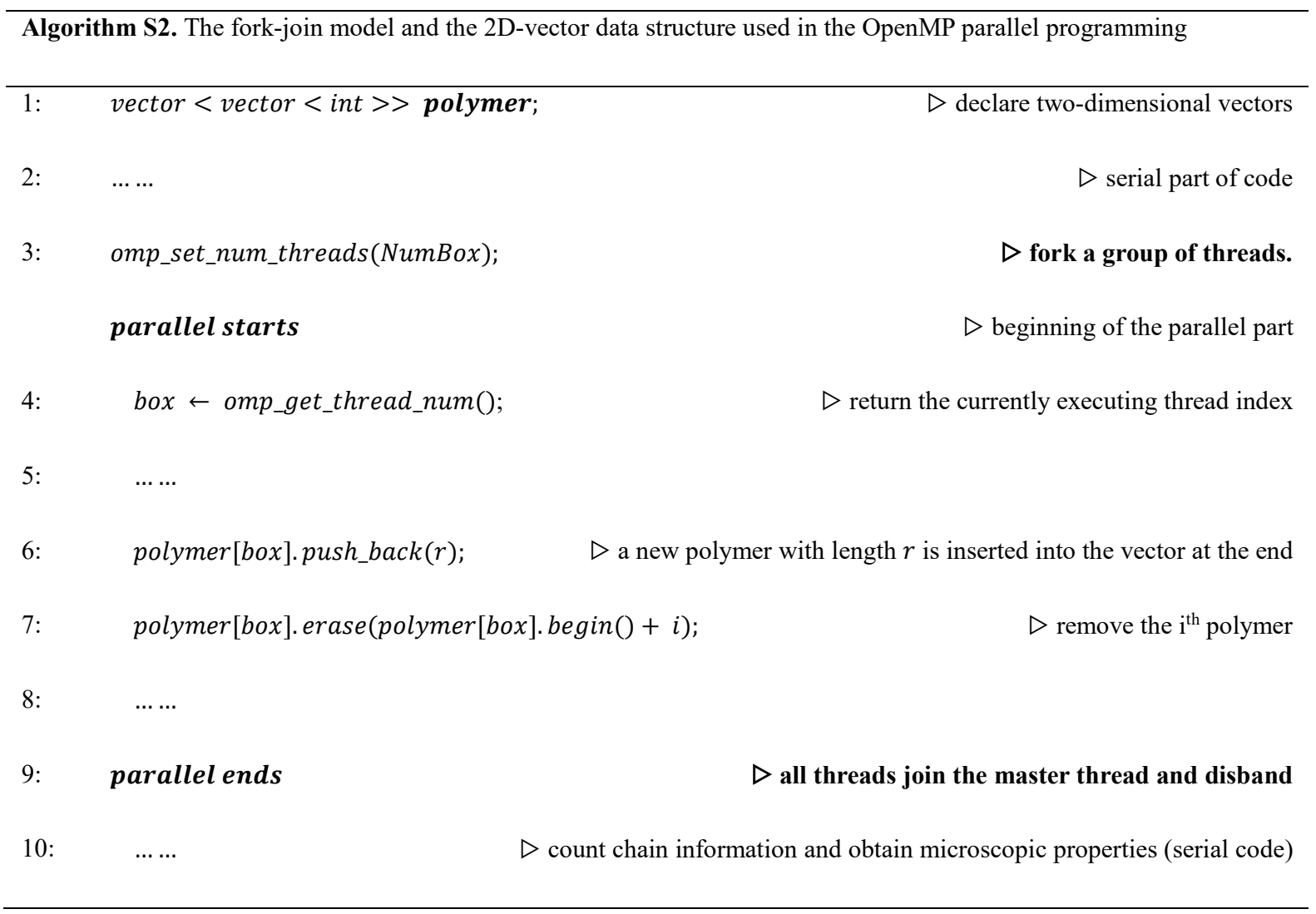


Algorithm S3. The random number generating and 1D array data structure on CUDA.

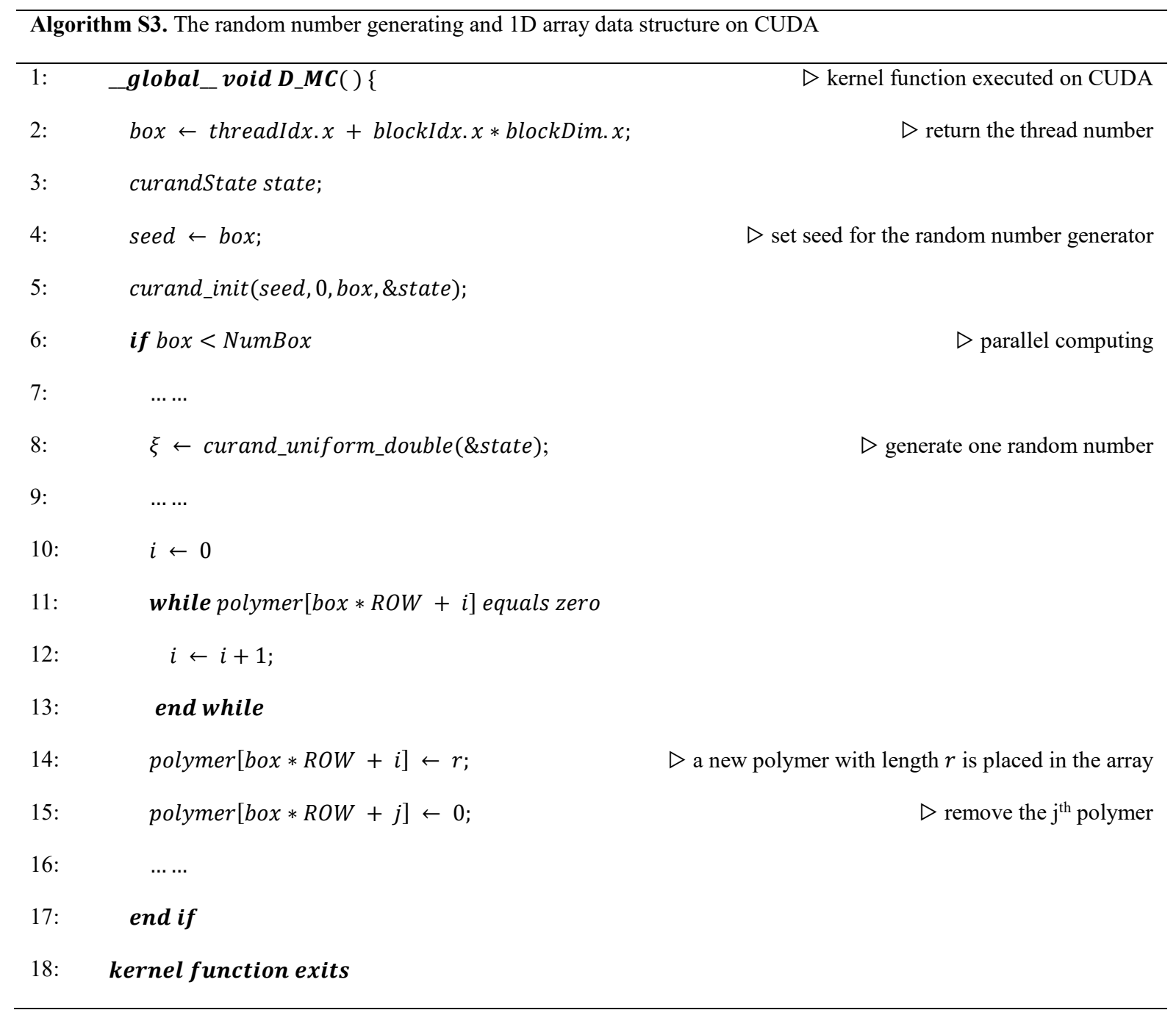


Algorithm S4. Return the suggested configuration for high parallel computing performance via function cudaOccupancyMaxPotentialBlockSize() and the invocations of the CUDA kernel function.

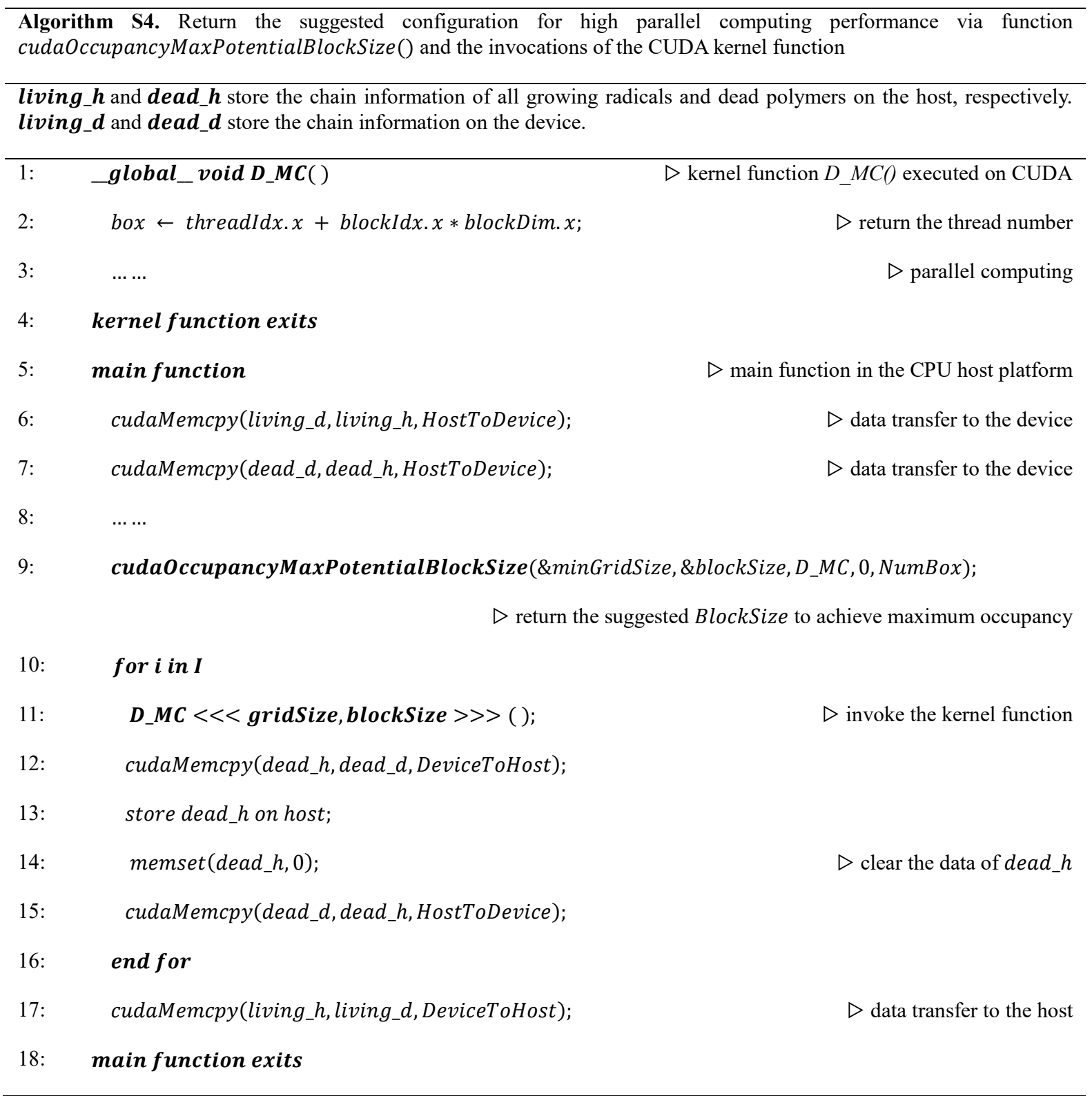




\section{Section C. Data Structure Details}

This section presents graphically the data structures and invocation methods for the two different parallelization procedures used. Figure S1, shows the 2D-vector data structure used in OpenMP parallel programming, with the first dimension indexing the sub-box number, and the second dimension indexing the polymer length in a sub-box. The corresponding methods for the 2D-vector are presented on the bottom panel. Figure S2, presents the 1D-array data structure used in CUDA parallel programming and the corresponding methods for the 1D-array.

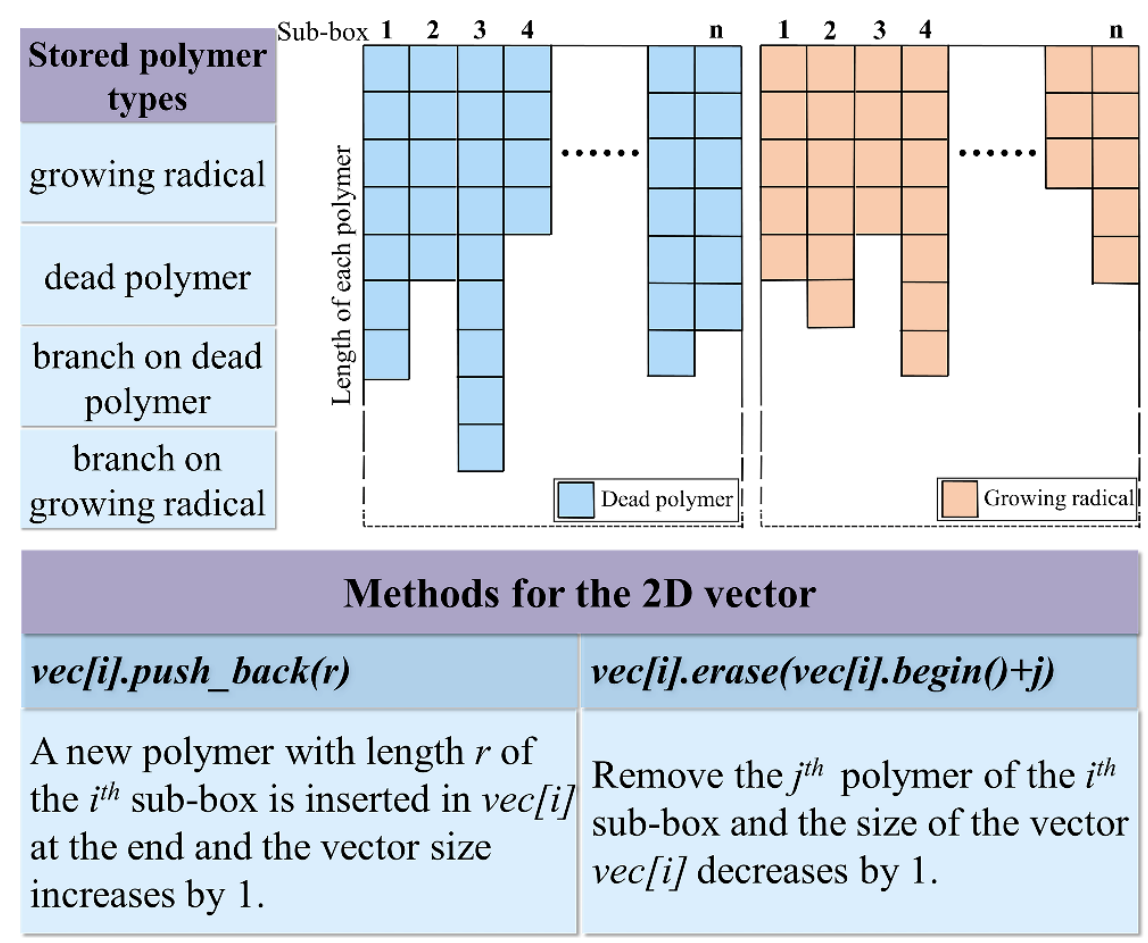

Figure S2. The data structure and corresponding methods to manipulate the data in twodimensional (2D) vectors in OpenMP programming on the CPU. 
Sub-box 1 Sub-box 2 Sub-box 3

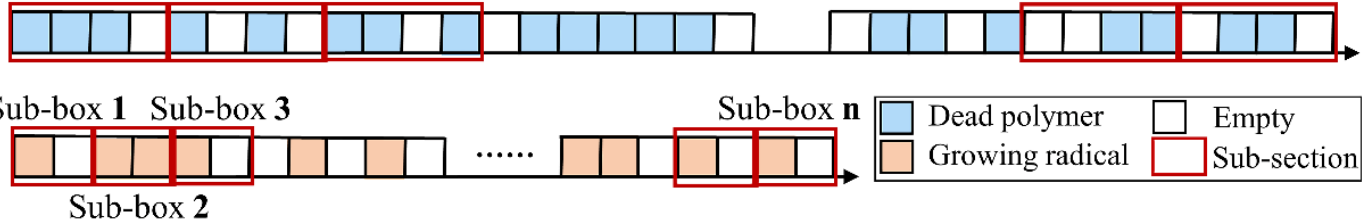

Sub-box 2

\begin{tabular}{|c|c|}
\hline \multicolumn{2}{|c|}{ Methods for the $1 D$ array } \\
\hline $\operatorname{ary}\left[i^{*} K\right][j]=r \quad(K$ is fixed $)$ & $\left.\operatorname{ary} / i^{*} K J / j\right]=0$ \\
\hline $\begin{array}{l}\text { A new polymer with length } r \text { of } \\
\text { the } i^{\text {th }} \text { sub-box is stored in } \\
\operatorname{ary}\left[i^{*} K\right][j] \text {. }\end{array}$ & $\begin{array}{l}\text { Remove the } j^{t h} \text { polymer of the } i^{t h} \\
\text { sub-box, by setting the value of } \\
\text { ary }\left[i^{*} K\right][j] \text { to zero. }\end{array}$ \\
\hline
\end{tabular}

Figure S3. One-dimensional (1D) array data structure and the corresponding methods to manipulate the data in one-dimensional arrays on CUDA. The stored polymer types are: growing radical, dead polymer, branch on dead polymer, branch on growing radical. 


\section{Section D. Sampling Method}

This section presents the sampling method of the DMC simulation. The quality of the random numbers $(r n d)$ generated in the sampling method directly affects the simulation results. The $10^{7}$ generated random numbers from $\mathcal{U}(0,1)$ through diverse random-number generators - function rand(), BOOST library, CURAND library on CUDA in the C language and function random.rand() in the Python language are counted, as shown in Figure S3. The interval $[0,1]$ is divided into $10^{6}$ equal-length bins and the random numbers fall into the corresponding bins. The conditional sampling method based on function $\operatorname{rand}()$ performed worst, and the other three random-number generators produced fairly high-quality random numbers. The average computing time of five different trials to produce $10^{7}$ random numbers from $\mathcal{U}(0,1)$ through four random-number generators are listed in Table $\mathbf{S 1 .}$

Table S1. Average Computing Time of Four Random-number Generators to Produce $10^{7}$ Random Numbers from $\mathcal{U}(0,1)$.

\begin{tabular}{ccc}
\hline random-number generator & language & average computing time (s) \\
\hline function rand () & $\mathrm{C} / \mathrm{C}++$ & 0.7314 \\
BOOST library & $\mathrm{C} / \mathrm{C}++$ & 1.1862 \\
CURAND library on CUDA & $\mathrm{C} / \mathrm{C}++$ & 0.0137 \\
function random.rand $)$ & Python & 5.6443 \\
\hline
\end{tabular}




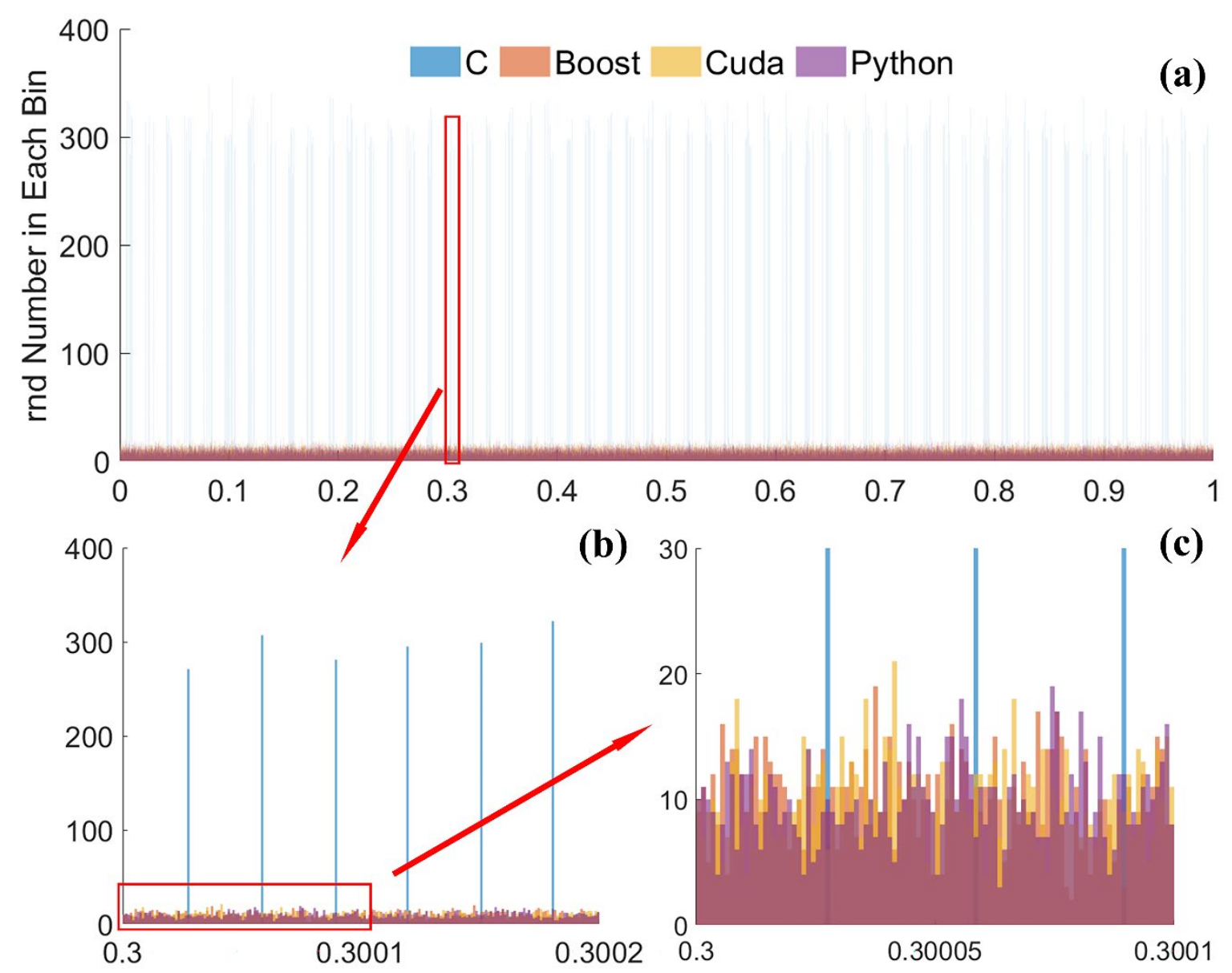

Figure S4. The histogram graph of the random numbers $(r n d)$ generated through function rand() (blue), BOOST library (orange), CURAND library on CUDA (yellow) in the C language, and function random.rand() in the Python language (purple), respectively. (a) The histogram graph of the random numbers in the interval $[0,1]$. (b) The histogram graph of the random numbers in the interval $[0.3,0.3002]$. (c) The enlarged picture of (b) with the vertical range in $[0,30]$. It is observed that function $\operatorname{rand}()$ falls short of following distribution $\mathcal{U}(0,1)$. 


\section{Section E. Simulation Metrics}

This section presents the simulation metrics for the DMC method of polymerization processes. Curve fitting is adopted to smooth the microstructure information for better presentation.

The DMC method for polymerization is able to give the polymer chain information at a given reporting time or conversion. The CLD and MWD are predicted after summarizing the chain information. The distributions are presented by a scatter plot. Curve fitting is introduced to facilitate the analysis, as shown in Figure S4. The horizontal axis is divided into multiple bins of size intervals and the average number of all points that fall in each bin is calculated. Points representing the average numbers are linked in turn to derive the curve plot. The horizontal axis is expressed as a set: $\left\{\operatorname{Bin}_{1}, \operatorname{Bin}_{2}, \ldots, \operatorname{Bin}_{N u m B i n}\right\}$, where NumBin denotes the number of bins. $\operatorname{Bin}_{b}(b=1, \ldots, N u m B i n)$ is an interval defined by:

$$
\operatorname{Bin}_{b}=\left(\left\lceil\frac{\text { Maximal chain length }}{\text { NumBin }}\right\rceil \times(b-1),\left\lceil\frac{\text { Maximal chain length }}{\text { NumBin }}\right\rceil \times b\right]
$$

The average of all points in the interval $\operatorname{Bin}_{b}$ is calculated by:

$$
w c_{b}=\frac{\sum_{k \in B_{i n}} Y(k)}{N o_{b}}(b=1, \ldots, \text { Nobin })
$$

where $N o_{b}$ is the number of points in the interval $\operatorname{Bin}_{b}$, and $Y(k)$ denotes the specific microscopic property value of point $k$.

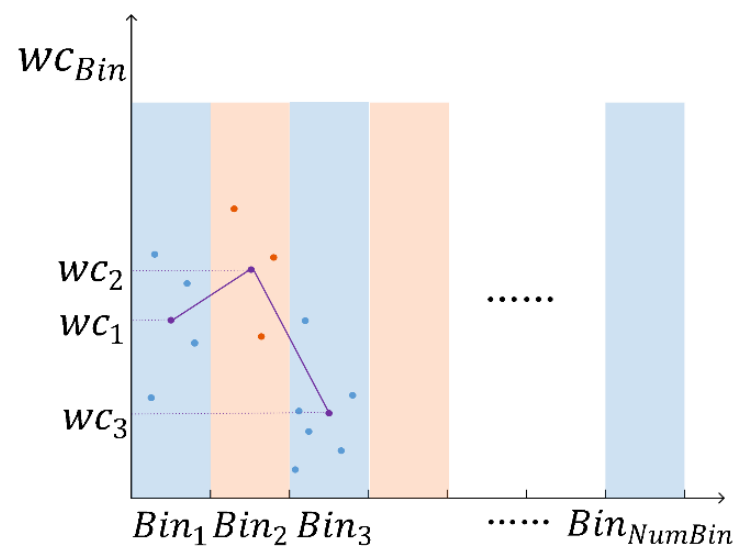

Figure S5. Curve fitting for the microscopic properties using the method of bins.

An inherent statistical error always exists in Monte Carlo based methods due to finite sampling points. According to the law of large numbers, the expectation value of the simulation results with sufficient particle numbers will converge to the expectation of an infinite-sized reaction system in the Monte Carlo method. The variance also decreases as the sampling points (the occurrence number of reaction events of the dynamic polymerization simulations) increase. 
Simulate an "undersized" reaction system i.e., one with a small molecular population many times, and the diverse random number sequences can result in different results. A too-small system size leads to erroneous simulation results with large expectation bias and variance. To meet the simulation accuracy requirement, the expectation of results should be convergent and the variance should be small. The simulation results from a "large" reaction system are accurate, but at the cost of a heavy computational burden. To balance accuracy and efficiency, it is necessary to find a proper system size. A valid method to measure the errors of simulation results is significant for the determination of a reaction system size. The self-distance and histogram distance proposed by Cao and Petzold [2] are adopted here to quantify the simulation result errors.

Suppose $A\left(A=\left\{a_{1}, a_{2}, \ldots \ldots a_{P}\right\}\right)$ and $B\left(B=\left\{b_{1}, b_{2}, \ldots \ldots b_{Q}\right\}\right)$ are two sets of sampled data with $P$ and $Q$ sampling points, respectively. The smallest and largest data points in the two sampling sets are:

$$
\begin{aligned}
& A B_{\text {min }}=\min \left\{a_{1}, a_{2}, \ldots \ldots a_{P}, b_{1}, b_{2}, \ldots \ldots b_{Q}\right\} \\
& A B_{\text {max }}=\max \left\{a_{1}, a_{2}, \ldots \ldots a_{P}, b_{1}, b_{2}, \ldots \ldots b_{Q}\right\}
\end{aligned}
$$

The interval $I=\left[A B_{\text {min }}, A B_{\text {max }}\right)$ is divided into $K$ subintervals $\left\{I_{1}, I_{2}, \ldots \ldots I_{K}\right\}$ with equal length. The range of the ith subinterval is:

$$
I_{i}=\left[A B_{\text {min }}+(i-1) \times \frac{A B_{\text {max }}-A B_{\text {min }}}{K}, A B_{\text {min }}+i \times \frac{A B_{\text {max }}-A B_{\text {min }}}{K}\right)
$$

The histogram distance is defined to measure the difference between two sample sets.

$$
\begin{gathered}
D_{K}(A, B)=\sum_{i=1}^{K}\left|\frac{\sum_{j=1}^{P} \chi\left(a_{j}, I_{i}\right)}{P}-\frac{\sum_{j=1}^{Q} \chi\left(b_{j}, I_{i}\right)}{Q}\right| \\
\chi\left(a_{j}, I_{i}\right)= \begin{cases}1, & a_{j} \in I_{i} \\
0, & \text { else }\end{cases}
\end{gathered}
$$

If sets $A$ and $B$ are obtained from the same system, the histogram distance defined as above is called the self-distance. The self-distance and histogram distance will be used to evaluate the simulation results in the next section. 


\section{Section F. Comparison with Deterministic Model Results}

The DMC simulation results of the reaction mechanism in Case 3 are checked against the deterministic method given in the $\operatorname{Ref}(1)$. To be consistent with the reference, $k_{T c}$ is set to $10^{6}$ and $k_{T d}$ is set to 0 here, and the other parameter values are the same as the ones in Table 10 of the manuscript. The MWD results of diverse conversions are compared as shown in Figure S6. The dash and solid curves denote the simulation results obtained from the parallel DMC method and the deterministic method, respectively. A good agreement between the two groups of results from the deterministic method and the sub-box parallel strategy is observed.

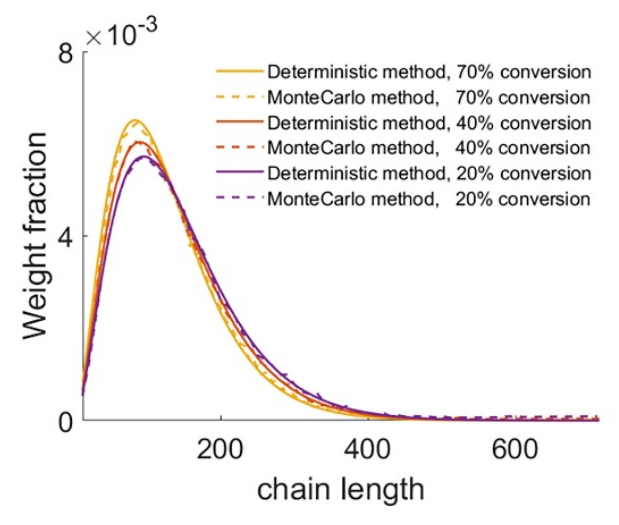

(a)

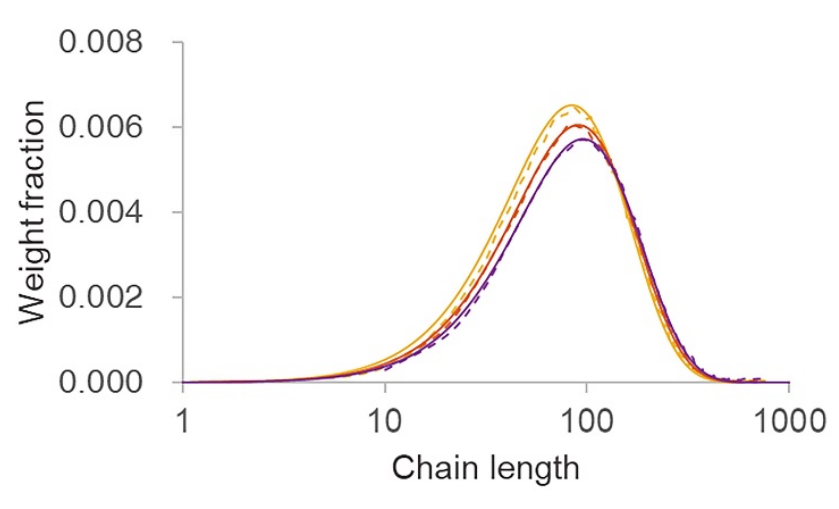

(b)

Figure S6. Comparison between the simulation results of the molecular weight distribution (MWD) as a function of the chain length for the stochastic and the deterministic models at different conversion values. (a) Plot in linear-linear scale. (b) Plot in linear-logarithmic scale. Good agreement between the two simulation methods is observed. 


\section{Section G. Calculation of Microscopic Rates}

The MC microscopic reaction rates of the five cases are listed in this section.

For Case 1, the MC microscopic reaction rates are listed in Table S2, where, $N_{X}$ denotes the number of molecules for reactant $X$, and $[X]$ denotes the concentration of reaction $X$.

Table S2. Monte Carlo (MC) Microscopic Reaction Rates for Case 1.

\begin{tabular}{cc}
\hline event & Monte Carlo microscopic reaction rate \\
\hline initiation & $R_{I}=k_{I}^{M C} \times N_{P_{0}} \times N_{M}=k_{I} \times[M] \times N_{P^{0}}$ \\
propagation & $R_{P}=k_{P}^{M C} \times N_{P} \times N_{M}=k_{P} \times[M] \times N_{P}$ \\
transfer to hydrogen & $R_{T H}=k_{T H}^{M C} \times N_{P} \times N_{H_{2}}=k_{T H} \times\left[H_{2}\right] \times N_{P}$ \\
\hline
\end{tabular}

The MC microscopic reaction rates for Case 2 are listed in Table S3.

Table S3. Monte Carlo (MC) Microscopic Reaction Rates for Case 2.

\begin{tabular}{cc}
\hline event & Monte Carlo microscopic reaction rate \\
\hline initiation & $R_{I}=k_{I}^{M C} \times N_{P_{0}} \times N_{M}=k_{I} \times[M] \times N_{P^{0}}$ \\
propagation & $R_{P}=k_{P}^{M C} \times N_{P} \times N_{M}=k_{P} \times[M] \times N_{P}$ \\
termination & $R_{T}=k_{T}^{M C} \times \frac{N_{P} \times\left(N_{P}-1\right)}{2}=\frac{k_{T}}{\Theta \cdot N A} \times N_{P} \times\left(N_{P}-1\right)$ \\
\hline
\end{tabular}

The MC microscopic reaction rates for Case 3 are listed in Table S4.

Table S4. Monte Carlo (MC) Microscopic Reaction Rates for Case 3.

\begin{tabular}{cc}
\hline event & Monte Carlo microscopic reaction rate \\
\hline initiation & $R_{I}=k_{I}^{M C} \times N_{I}=k_{I} \times N_{I}$ \\
propagation & $R_{P}=k_{P}^{M C} \times N_{P} \times N_{M}=\frac{k_{P}}{\Theta \cdot N A} \times N_{P} \times N_{M}$ \\
termination by combination & $R_{T c}=k_{T C}^{M C} \times \frac{N_{P} \times\left(N_{P}-1\right)}{2}=\frac{k_{T c}}{\Theta \cdot N A} \times N_{P} \times\left(N_{P}-1\right)$ \\
termination by disproportionation & $R_{T d}=k_{T d}^{M C} \times \frac{N_{P} \times\left(N_{P}-1\right)}{2}=\frac{k_{T d}}{\Theta \cdot N A} \times N_{P} \times\left(N_{P}-1\right)$ \\
\hline
\end{tabular}


The MC microscopic reaction rates for Case 4 are listed in Table S5.

Table S5. Monte Carlo (MC) Microscopic Reaction Rates for Case 4.

\begin{tabular}{cc}
\hline event & Monte Carlo microscopic reaction rate \\
\hline initiation & $R_{I}=k_{I}^{M C} \times N_{I}=k_{I} \times N_{I}$ \\
propagation & $R_{P}=k_{P}^{M C} \times N_{P} \times N_{M}=\frac{k_{P}}{\Theta \cdot N A} \times N_{P} \times N_{M}$ \\
chain transfer & $R_{T r}=k_{T r}^{M C} \times N_{P} \times N_{M}=\frac{k_{T r}}{\Theta \cdot N A} \times N_{P} \times N_{M}$ \\
termination by combination & $R_{T c}=k_{T c}^{M C} \times \frac{N_{P} \times\left(N_{P}-1\right)}{2}=\frac{k_{T c}}{\Theta \cdot N A} \times N_{P} \times\left(N_{P}-1\right)$ \\
termination by disproportionation & $R_{T d}=k_{T d}^{M C} \times \frac{N_{P} \times\left(N_{P}-1\right)}{2}=\frac{k_{T d}}{\Theta \cdot N A} \times N_{P} \times\left(N_{P}-1\right)$ \\
\hline
\end{tabular}

The MC microscopic reaction rates for Case 5 are listed in Table S6, where $r$ denotes the chain length, and $N_{D, r}$ represents the number of dead polymers with length $r$. The chain length here represents the monomer unit number of a polymer.

Table S6. Monte Carlo (MC) Microscopic Reaction Rates for Case 5.

\begin{tabular}{cc}
\hline event & Monte Carlo microscopic reaction rate \\
\hline initiation & $R_{I}=k_{I}^{M C} \times N_{I}=k_{I} \times N_{I}$ \\
propagation & $R_{P}=k_{P}^{M C} \times N_{P} \times N_{M}=\frac{k_{P}}{\Theta \cdot N A} \times N_{P} \times N_{M}$ \\
chain transfer & $R_{T r}=k_{T r}^{M C} \times N_{P} \times \sum_{r=1}^{\max (r)} r \times N_{D, r}=\frac{k_{T c}}{\Theta \cdot N A} \times N_{P} \times \sum_{r=1}^{\max (r)} r \times N_{D, r}$ \\
termination by combination & $R_{T c}=k_{T C}^{M C} \times \frac{N_{P} \times\left(N_{P}-1\right)}{2}=\frac{k_{T c}}{\Theta \cdot N A} \times N_{P} \times\left(N_{P}-1\right)$ \\
termination by disproportionation & $R_{T d}=k_{T d}^{M C} \times \frac{N_{P} \times\left(N_{P}-1\right)}{2}=\frac{k_{T d}}{\Theta \cdot N A} \times N_{P} \times\left(N_{P}-1\right)$ \\
\hline
\end{tabular}




\section{REFERENCES}

(1) Lu, J.; Zhang, H.; Yang, Y. Monte Carlo Simulation of Kinetics and Chain - length Distribution in Radical Polymerization. Macromol. Theory Simulations 1993, 2 (5), 747 760.

(2) Cao, Y.; Petzold, L. Accuracy Limitations and the Measurement of Errors in the Stochastic Simulation of Chemically Reacting Systems. J. Comput. Phys. 2006, 212, 6-24. 